\title{
Conclusions: localizing global sport for development
}

In this chapter we draw together the main themes which have emerged from our research in Zambia, and reflect critically on how they may contribute to the overall aspiration for this book: to 'localize' global SfD. We are equally concerned with the knowledge and understanding that our empirical work may offer and with the research processes that have underpinned it. We first consider the extent to which our findings may have brought to the fore features of SfD that have previously been underexplored in the wider literature on 'global' SfD. This discussion is arranged around three overarching themes that emerged across the empirical chapters. Firstly, focusing on multifaceted SfD practices and experiences, we explore the diversity and complexity of $\mathrm{SfD}$ as practised and experienced in Zambia; secondly, we consider associations between SfD and 'mainstream development', examining relationships between SfD and other development sectors in Zambia; and thirdly, we confront the central issue of local to global, reviewing the book's evidence on how SfD interacts with the multiple contexts in which it occurs. In the fourth and final section of the chapter we then consider the research process through which these findings were obtained, and offer some reflections on wider knowledge-production approaches across SfD.

To frame these conclusions, it is useful to reprise how the book sits within the wider landscape of SfD literature. Since we first embarked on it, the volume of published research associated with SfD has undoubtedly burgeoned, with two approaches remaining dominant. The first of these is the 'evidence' orientation, prominent within both SfD policymaking and academic communities. The evidence discourse underpins the extensive body of evaluation research conducted in SfD, and prioritizes forms of enquiry intended to have instrumental value for improving SfD policy and practice, including those with the capacity to identify causal links to (measurable) outcomes. Similar instrumental aspirations underpin the associated development of decontextualized 'models' of SfD that purport to inform policy and practice, as well as the evaluation of specific programmes. 
The second prominent strand in the SfD literature is that of critical-theoretical approaches that seek to locate SfD within the broader political, economic and social structures commonly associated with postcolonialism. Although these approaches can enable examination of local contexts and local agency (Darnell and Hayhurst, 2012), to date they have largely been utilized to explore internationally orientated aspects of SfD (e.g. Darnell, 2007; Hayhurst, 2009; Forde, 2014). Through this, the 'local' becomes understandable through the lens of global and international structures. This approach to the local is also evident in some empirical studies which have not specifically adopted the postcolonial theoretical position; although utilizing data collected in African localities, accounts by Straume and Hasselgård (2014; also Hasselgård and Straume, 2015) and Guest (2009) have also placed Northern-instigated SfD programmes in the context of postcolonial relations before examining the responses, including resistance, of local stakeholders. The smaller number of authors who have taken local dimensions of SfD as their focus (e.g. Armstrong, 2004; Fokwang, 2009; Kath and van Buuren, 2014) have been constrained in their investigation of the multidimensional relationships between these local contexts and broader political, economic and social structures.

This book sits in a rather different space from these two approaches. It differs substantially from evidence-oriented studies in $\mathrm{SfD}$, and in more nuanced yet significant ways from most critical postcolonial writings. The book's position has evolved organically as our knowledge of SfD in Zambia has grown, and the contours of the (academic) SfD field became more clearly demarcated. The theoretical, methodological and empirical strands of development studies literature have also been influential, most notably Norman Long's (2001) writings on actor-orientated sociology. This advocates developing more differentiated and contextualized understandings of development work by grounding these in the perspectives and experiences of multiple stakeholders, then locating these 'local' perspectives within broader political, economic and social-structures contexts. This reflects our own strategy in the book's empirically based chapters, which bring together perspectives from a range of stakeholders and from across different, distinct research projects. Again following Long (2001), we have also used diverse literatures and concepts throughout the book to help understand these local perspectives in light of broader aspects of both sport and international development. In the following sections, these ingredients are now brought together to examine, in turn, multifaceted practices and experiences of Zambian $\mathrm{SfD}$, associations between SfD and other development sectors, and relationships between local dimensions of SfD and broader influences. 


\section{Multifaceted SfD practices and experiences}

First and foremost, findings from across the research reveal that SfD practices and experiences in Zambia are characterized by diversity and complexity. While this is not the most surprising conclusion to arise from multiple studies over an extended time period, it does reveal different aspects that repay attention. Firstly, the diversity and complexity revealed in Zambia add to debates that consider how the characteristics and functions of SfD have been classified; secondly, identification of complexity and local specificity challenges some of the more instrumental approaches for SfD put forward by external stakeholders - mostly from the global North - interested in the utilization of tightly defined 'models' to support evaluation and develop practice.

The first of these points concerns the questions that our findings raise about how the relationships between sport and aspects of 'development' are conceptualized within SfD. As we noted in Chapter 1, throughout the SfD literature efforts have been made to address the different ways in which 'development of' and 'development through' sport take place. Terminology such as Coalter's 'sport plus' and 'plus sport' has been used in a variety of ways - for example, to classify organizations (e.g. Giulianotti, 2011a), to distinguish between the aims of sport programmes (e.g. Coakley, 2011) or the relative emphasis given to sport within them (e.g. Coalter, 2010b: 298), and assert the likelihood of sport programmes contributing to developmental outcomes (Hartmann and Kwauk, 2011). Notwithstanding their wide use, such distinctions have not been clear-cut, as their authors admit, and our own findings concur with this.

The multilayered nature of our research in Zambia offers some additional insights into the complex picture that arises from interrelated and mutually constitutive practices in sport and SfD. At policy level, the historical analysis in Chapter 2 has shown how closely the emergence of the SfD sector in Zambia has been interwoven with the broader development of sport policies and provision. This is also observable at the organizational level, where the major contributions to development of sport provision, activities and facilities made by two NGOs, Sport in Action and EduSport, supplement and complement their more commonly recognized 'development through sport' endeavours. At programme level, single strategies can cut across multiple objectives, as in the use of peer leadership within SfD programmes, which simultaneously increases provision of sport opportunities and fosters personal and social development. The research has also uncovered examples of elements of SfD work that extend beyond conventional sport practices, such as the internships offered by EduSport, which 
are more resonant of youth- and community-development activities than mainstream sport provision. All of these examples suggest that understandings of SfD may be limited by overly narrow and rigid distinctions.

Rigid distinctions between development 'of' and 'through' sport also risk underplaying aspects of young people's experiences of $\mathrm{SfD}$, which can draw on either or both. As discussed in Chapter 6, many young Zambians placed value on simply participating in organized and competitive sport activities developed by SfD NGOs, with some then also deriving what they considered to be wider benefits as a result. Young people's experiences, therefore, cut across and were mutually reinforced by multiple aspects of SfD in ways which defy clear-cut analytical distinctions. This compounded response is not wholly accounted for through conceptual devices such as 'balancing' the different elements (Hartmann and Kwauk, 2011) within SfD activities, nor by placing them on a 'continuum of sport plus and plus sport' approaches (Coalter, 2010b: 298); rather, the different aspects and experiences of local SfD are instead intertwined and mutually constitutive. The complexity evident in Zambia reinforces the argument that at many levels of SfD, boundaries between aims, approaches and activities are not clearly defined, and understanding of SfD work is not necessarily enhanced by imposing such delineation.

A second issue that arises from the complex and multifaceted nature of SfD in Zambia concerns the resultant implications for efforts within SfD to offer normative prescriptions for practice. As well as contributions in the instrumental strand of the SfD literature, numerous policy documents, manuals and toolkits, such as those held on the International Platform for Sport for Development and Peace, present 'guidelines', 'curricula' and illustrative examples of 'best practice' for SfD (e.g. SDPIWG, 2008b; Commonwealth Secretariat, 2014). Critical scholars (e.g. Hayhurst, 2009; Forde, 2014) have identified how the discourses represented in such documents and curricula reinforce, or at least fail to challenge, hierarchies of power that contribute to the subjugation of those involved in $\mathrm{SfD}$ in the global South. The picture emerging from Zambia suggests that, notwithstanding this global context, the local practices of SfD organizations may themselves offer a counterpoint to normative prescriptions.

This is evident in the elements of both pragmatism and intentionality that occur in the practices of Zambian SfD organizations, such as choices made regarding the delivery of particular sports. Some Zambian SfD organizations, for example, have chosen to develop women's football specifically to confront and resist local gender norms that contribute to inequality. Conversely, on other occasions, they also encourage separate male and female participation in 
competitive football and netball respectively. While these sports may be representative of colonial influences (as well as locally held gendered beliefs), their adoption is also indicative of a degree of pragmatism on behalf of SfD organizations, as the delivery of football and netball is popular with young people and requires relatively few material resources. That both pragmatism and intentionality informs the decision making of Zambian SfD organizations stands in contrast with normative prescriptions that support planning on the basis of largely instrumental choices regarding the achievement of desired outcomes.

Another feature of such prescriptions is that they either implicitly (e.g. Higgs, 2014) or explicitly (e.g. Gannett et al., 2014) promote more formalized planning and decision making by $\mathrm{SfD}$ organizations. This contrasts with the many instances of informality we have noted in Zambian SfD, in areas such as the selection, training and progression of peer leaders; the development of relationships between SfD participants, deliverers and other community stakeholders; and in some partnership working between different organizations, particularly at more localized levels. More importantly, just as with some pragmatically orientated decisions, aspects of informality reflect aspects of the localized cultural and material contexts within which SfD in Zambia exists. This is not to suggest that 'informal' approaches to SfD are in any sense objectively 'better' than formal ones, but rather that in any specific context, informality may be locally appropriate, and attempts to enforce more 'formalized' management potentially detrimental. Although the importance of adaptability to local context is recognized in instrumental SfD literature (e.g. Lyras and Welty Peachey, 2011; Schulenkorf, 2012), it is inevitable that overarching models downplay local contextual possibilities, influences and constraints on SfD.

One of the prominent normative features in international policies, international donors' practices and instrumental academic SfD literature is an insistence that desired outcomes of SfD programmes should be specified more precisely. For example, in guidance claimed to be developed from Kay and Dudfield's (2013) evidence-based review, Higgs states that 'detailed programme objectives need to be developed. These should be concise statements that state who will make what change, by how much, where and by when' (Higgs, 2014: 16 ; italics as in original). Coalter's (2013) research is aligned with this approach in seeking to evaluate changes in specific and narrow measures of young people's self-esteem and self-efficacy over the time of their involvement in particular SfD programmes. However, if the orientation towards tightly defined SfD programmes and outcomes is redirected towards foregrounding young people's experiences of engagement with $\mathrm{SfD}$, then a rather more complex picture 
emerges. As Chapter 6 indicated, young Zambians participating in activities provided by EduSport and Sport in Action not only experienced but actively sought different outcomes from their engagement with SfD. Furthermore, the types of benefits identified by young people ranged from those directly resulting from participating in sport to various dimensions of personal development advanced through both sport activities and further non-participatory opportunities developed by the same SfD organizations. These varied experiences and responses of young people are themselves reflective of the diversity of approaches enacted by Zambian SfD, although no particular claim to causality is made here.

A further notable aspect of Zambian SfD is the importance of the communitarian environment associated with sporting activities and the organizations that develop them. As discussed in Chapters 5 and 6, the development of such a communitarian environment by Zambian SfD organizations reflected both long-standing aspects of local culture and the concerns voiced by many Zambians regarding the loss of networks of family and community support. Most studies of specific SfD programmes have focused on the personal benefits for young people individually - benefits which our young Zambian interviewees also report. However, this focus on single programmes and specific, mainly individualized, outcomes may have limited exploration of the interweaving of various $\mathrm{SfD}$ practices, the significance of their local context, and the potential collective benefits that can also accrue, as identified in Zambia.

Peer leaders especially identified with the communitarian ethos of their SfD NGOs. There is an element of circularity in that peer leaders personally drew benefits from their relationships in and with SfD NGOs, and this in turn helped them to provide appropriate role models for other young people. Such a finding leads to questions about models developed to explore how $\mathrm{SfD}$ processes may produce outcomes. Approaches to modelling SfD tend to suggest a sequential relationship between the 'impacts of participation on individuals, and the resultant outcomes' at broader community levels (Coalter, 2014: 72). Sugden's (2011, 2014) 'ripple' model, for example, suggests that benefits for individual young people participating in sports activities may be an antecedent to broader changes at different levels of society. However, for SfD organizations in Zambia, prior work has been necessary to change community, and particularly parental, attitudes towards sport before young people - especially girls - are even allowed to participate in activities, let alone gain any personal benefit from doing so. This example alone suggests that the assumption that individual benefits aggregate to collective ones is somewhat simplistic and points to the limitations of approaches to modelling SfD. 
A normative impetus towards sequential modelling of SfD is nonetheless dominant both in international agendas for SfD organizations and in approaches to evaluating SfD programmes. In both, there is widespread advocacy for the use of programme theories or logic models (Levermore, 2011). While this does not wholly exclude non-linear causality between SfD processes and outcomes, it unquestionably involves assumptions about the sequential arrangement of SfD mechanisms and their effects (e.g. Coalter, 2013). Yet, as is evident throughout this book, simplified programme theories or logical models cannot capture the diversity and complexity of Zambian SfD. The danger of utilizing many of the models and frameworks suggested for SfD is that they inherently obscure the important complexities and multifaceted aspects of SfD that locally oriented studies reveal. The recent reflection on programme theories and logic models by Brian Pratt, long-time international development practitioner and editor of the journal Development in Practice, is particularly apposite: 'one is left asking whether these methods relate to reality or merely the imagination of development planners' (Pratt, 2015: 144). Different methodological approaches to those currently prominent in SfD are required to ensure that more localized, nuanced and 'real' understandings emerge.

\section{Associations between SfD and 'mainstream' development}

The second area in which our research may contribute to debates in SfD is in discussions about the relationship between SfD and so-called 'mainstream' development. One of the recurrent calls from within SfD is for closer partnership between SfD and the much longer established development field. In reality, 'development' is of course an extensive, diverse and dynamic area, characterized by divergent standpoints: to speak of a singular 'mainstream' is therefore misleading. Nevertheless, the purpose of this section is to consider what our research in Zambia contributes to knowledge about associations between SfD and other development sectors, in policy, practice and research.

In conducting our research we have very much aligned with Darnell and Black's (2011: 370) concern that 'insights from development studies often appear underappreciated in SDP [sport for development and peace] circles'. Like other SfD researchers who have utilized critical theories from development studies, our own approach has been to draw on a range of development studies literature and research. Our particular contribution is through the empirical examination of relationships between SfD and other development sectors, which remains relatively rare and may allow the more generalized conclusions that have so far 
been available (e.g. Darnell and Black, 2011; Giulianotti, 2011b) to be further developed.

The association between the establishment of SfD in Zambia and broader trends of national governance and global neo-liberal ideologies was examined in Chapter 2. This showed how, in its initial phases, $\mathrm{SfD}$ emerged to address gaps in state provision, with the prominence of NGOs reflecting trends in development approaches nationally and globally. Subsequently, however, there has been divergence; the $\mathrm{SfD}$ sector has yet to reform (or be reformed) in line with significant changes in development governance more broadly. While there are relatively few empirical studies from Zambia, there has been global impetus from early in the twenty-first century towards more co-ordinated state- and country-led approaches in other development sectors, even if these ambitions have not been fully realized in practice. As yet, there is limited evidence of these trends being implemented in Zambian SfD and any rhetorical commitment to do so is relatively weak.

Aspects of divergence between $\mathrm{SfD}$ and other development sectors were also very evident in Chapter 3, where the relationship between Zambian SfD and HIV/AIDS organizations and policies was examined. This revealed very little integration of $\mathrm{SfD}$, either generally or in terms of particular organizations, in nationally instituted HIV/AIDS policies and structures. SfD appeared subject to a degree of institutionalized marginalization arising from multiple factors, including a lack of collective advocacy on behalf of the SfD sector, and ingrained beliefs held by HIV/AIDS stakeholders which undervalued SfD practice. There was also incompatibility as a result of a lack of alignment in approaches to monitoring and evaluation, which had been initiated by different international agencies and donors in both the SfD and HIV/AIDS sectors.

This national picture of sport being a relatively peripheral concern within development policy does not necessarily hold at other levels of analysis. Certainly, in Zambia, our localized data revealed rather different findings about the intersections of SfD and development actors at community level. SfD NGOs were often at the forefront of developing co-ordinated approaches amongst different organizations working with young people in some local communities. Many community-based representatives of development organizations spoke favourably of sport, and valued it as an attractive form of provision for young people for whom there were few low-cost structured activities available in school, community or church contexts. Although these institutions were important in the lives of young Zambian people, several stakeholders saw value in 
sport precisely because it offered an alternative to these established and traditional environments.

For local stakeholders, sport could therefore be important in its own right, as a relatively new, more neutral and less culturally constrained context for development than established institutions. To some extent this runs counter to views that advocacy for SfD is 'evangelistic' (Coakley, 2011; Coalter, 2013) - a label increasingly applied to any positive expressions about the capacity of sport to contribute to 'development'. In our Zambian studies, and as Kay (2009) also found in Delhi, India, it was notable that positive local views of sport were expressed by interviewees from outwith the SfD sector, individuals with little personal stake in it. Critical analyses that focus on the historically inscribed nature of sport at the macro level, including expert accounts of colonialism (e.g. Darnell and Hayhurst, 2012), perhaps underplay the possibility of differentiated local understandings. In order to enhance future investigation, it is useful to recognize the possibility that pivotal contributions to SfD scholarship risk obscuring ways in which local perspectives on sport may inform communitybased approaches, practices and relationships.

Another area in which the practices of SfD and development organizations are aligned is through their work with peer leaders. As noted above, peerleadership systems are enacted by Zambian SfD organizations in diverse ways, and peer leaders themselves are not homogeneous in either their personal characteristics or the SfD roles they fulfil. Despite the prominence of peer leadership within SfD, this complexity has not been the focus of SfD scholarship, which has instead mainly focused on the benefits peer-led activities deliver for participants (Maro et al., 2009; Woodcock et al., 2012). The wider development literature on peer leadership in sub-Saharan Africa offers a little more recognition of the experiences of peer leaders (e.g. Molassiotis et al., 2004; Svenson and Burke, 2005; Lesko, 2007), However, research has rarely had a significant focus on peer leaders themselves. Given the importance of peer leaders to the delivery of SfD and development activities, further investigation into the varied perspectives and experiences of peer leaders seems likely to have value to improved understanding in both of these fields.

A final consideration is how young people may themselves experience intersections between SfD and other development sectors. Our research has identified some ways in which this may occur; for example, many SfD peer leaders also fulfil similar roles in other development-orientated activities outside SfD, and many SfD participants receive development content such as HIV/AIDS education in a number of different contexts. The research has also found 
examples of SfD activities providing a context in which young people discuss information gained from other environments, which suggests a degree of flexibility in educational SfD activities that can be lacking from some other HIV/ AIDS interventions in Zambia (see Chapter 4). Our data on this aspect is, however, somewhat limited and does not allow full examination of how young people experience the dissemination of messages about HIV/AIDS from diverse sources, which may be different, potentially conflicting or partial in their content. This suggests that research that seeks to identify a narrow 'SfD effect' in terms of improvements in HIV/AIDS knowledge needs to take into account the ways in which differently gained knowledges intersect and relate to one another. Such intersections are more likely to be understood through research that methodologically foregrounds young people rather than studies that focus on specific programmes, either individually or comparatively.

In concluding this section we risk being repetitive by reasserting that the associations between $\mathrm{SfD}$ and 'mainstream development' are characterized by complexity and diversity. Nevertheless, this is the inevitable conclusion from our research at different levels and across different sectors. Broadly speaking, the level of integration between $\mathrm{SfD}$ and other development sectors appears to be greater and more complementary as analysis moves from national to local (community) levels, as well as from contexts which are more structured to those with greater scope for stakeholder agency. Although some of our further research beyond Zambia (Lindsey et al., 2014) suggests that similar patterns may occur in other countries where $\mathrm{SfD}$ has marginal policy status, we cannot assert this; as in Zambia, such observations require a wide range of research across the levels and sectors of development within single countries, as well as thorough engagement with relevant development studies literature. Following on from this differentiated analysis, we now attempt closer scrutiny of the ways in which $\mathrm{SfD}$ in Zambia has been subject to influences from the global to the local.

\section{From local to global: influential and influencing SfD contexts}

The final set of conclusions that we draw from our research concerns the central issue that the book addresses - the relationship between local and global SfD. In addressing this issue, we recognize that global and local are not dichotomous dimensions, nor is either singular. This has been clearly evident in Zambia, where SfD occurs within contexts which are both multidimensional and multilayered. Our data is too limited to reveal the full complexity of these contexts; 
for example, our findings concerning the limited integration of SfD with the HIV/AIDS sector in Zambia may or may not be applicable to relationships between SfD and other sectors, such as education. Similarly, we cannot offer comparative analysis of SfD in different Lusaka communities, let alone across rural and urban areas. Nonetheless, our findings provide some insights into the diverse ways in which SfD in Zambia reflects, influences and is influenced by the various contexts in which it is situated. The section examines the development issues to which SfD activities in Zambia are orientated and to which these activities may, potentially, contribute, and pays particular attention to how locally specific and broader contextual influences interact to affect them.

One of the concerns expressed about SfD is the limited extent to which it can offer solutions to what Coalter (2010b) terms 'broad-gauge' social problems. Nevertheless, our research indicates that the multifaceted orientation and delivery of SfD can reflect local understandings of interconnected social problems. For example, the strongly communitarian ethos of Sport in Action and EduSport is a response to local concerns regarding the breakdown of familial and social support systems as a result of the HIV/AIDS pandemic. More generally, the deep and nuanced recognition of local social and structural problems by adults and young people involved with SfD suggests a more informed and constructive framing than the view that SfD positions young people as in need of 'development' because they are considered to be 'deficient' in some respects (Coalter, 2014).

Expanding on these points, our research indicates that Zambian SfD organizations are often able to exert a considerable degree of agency. This contrasts with the emphasis given in the global SfD literature to the levers of power utilized by Northern donors (e.g. Levermore, 2009; Nicholls et al., 2011; Kay, 2012). While the impacts of funding and accountability processes are unquestionably evident in Zambian SfD as elsewhere, so too is the agency of Zambian NGOs as they both shape SfD according to local contexts and limit international impetus towards more precisely defined objectives for SfD. While Northern donor organizations may significantly influence particular funded programmes, the overall ethos and approaches of the two Zambian SfD NGOs that we have worked with most closely shows significant consistency over time with the principles on which they were initially founded.

There are also indications that the NGOs' capacity to resist and reshape donors' influence has developed and increased over time. This resonates with the view expressed by Bob Munro (reported in Coalter, 2010a), the founder of the MYSA in Nairobi, who observed that by initially developing without the 
support of international partners, the organization was subsequently able to gain agency. In something of a contrast, agency amongst Zambian SfD NGOs appeared to increase as they gained experience working with Northern donor organizations. As discussed in Chapter 3, this degree of agency was partly enabled by factors that limited Northern donors' ability to exert influence over Zambian SfD organizations. Both collectively and individually, many Northern $\mathrm{SfD}$ donor organizations and their staff were inexperienced in the processes of international development generally and required time to become well acquainted with local SfD practices specifically. The changeable approach by Norwegian donors to funding SfD in Zambia (identified in Chapter 2) is another indication of Northern funders' ongoing process of adaption to the contours of international development (Straume and Hasselgård, 2014).

Overall, therefore, our findings suggest that there has been more scope for local agency among Zambian SfD stakeholders than the global SfD literature suggests. This is not to say that such local agency is not constrained in other ways, by other contextual influences. For example, one of the common challenges faced by Zambian SfD organizations was overcoming the widely held view that sport was 'just play' and lacks developmental significance. This perspective had consequences at multiple levels, from inhibiting the integration of these organizations into national HIV/AIDS structures to discouraging parents from allowing young people, especially girls, to participate in community SfD activities. Further empirical research into the influences of local social contexts on $\mathrm{SfD}$ is needed to ascertain whether similar issues are encountered by $\mathrm{SfD}$ organizations delivering activities cross other African countries.

Similarly, detailed empirical examination can improve understanding of the ways in which potential and actual outcomes of SfD are influenced by contextual conditions. In Chapter 6, we identified a number of ways in which the benefits young people obtain from their engagement in SfD may be defined or constrained by the contexts within which they find themselves. Some young people talked about sport activities as something of a palliative to the conditions of their everyday lives; others, especially young females, spoke of their struggles and inability to overcome traditional Zambian social hierarchies and other structural constraints. Individual young people clearly perceive and experience their social contexts differently, and differ in how they orientate their responses both to these social contexts and to SfD.

When the focus shifts away from immediate local contexts and SfD is considered in relation to global social and economic conditions, further influences on the multifaceted relationship between structure and agency at local level are 
evident. Here, too, they lead to differentiated conclusions. On the one hand, the overarching communitarian ethos of Zambian SfD organizations sits in contrast to neo-liberal individualism; on the other, the emergence of Zambian SfD NGOs is directly associated with the global impact of neo-liberal development policies. Similarly, the orientation of Zambian SfD to addressing HIV/AIDS has been important in countering the localized consequences of the pandemic, but also reflective of the propagation of HIV/AIDS through deeply rooted global inequalities that are sustained by hegemonic power relations (UNAIDS, 2008). The global context in which Zambian SfD is located unquestionably exerts significant influence on it - yet alongside this, and within Zambian communities, practices are also locally orientated, defined, challenged and experienced.

Local dimensions have not been conceptually ignored in the SfD literature, but have seldom been the focus of detailed empirical investigation. This risks fostering an overall understanding of SfD that is collectively coherent but relatively undifferentiated. In the case of Zambia, broad historical and global inequalities have undoubtedly shaped the political and social context in which SfD has emerged; however, we can also question the extent to which international relationships specific to SfD have defined the organizations, practices, activities and outcomes of Zambian SfD. Thus, rather than local and global dimensions being singular, or international SfD being indivisible from broader global inequalities, a more complex picture emerges of a mosaic of multiple interconnected locals and globals, each with diffuse boundaries. Recognition of this complexity and fluidity offers new dimensions to understanding of the globallocal phenomenon of SfD.

\section{Developing understanding and contributing to knowledge production: reflections on localizing SfD through research}

The preceding sections sought to distil the substantive findings of our research. Three conclusions emerged: that SfD practices and experiences in Zambia are complex and multifaceted; that there are differentiated associations between SfD and 'mainstream development', with closer integration sometimes occurring at local level where scope for stakeholder agency is greatest; and that the interplay of 'global' and 'local' produces complex, differentiated and, above all, locally specific manifestations of SfD. This ability to detect and present the very particular nature of local SfD practices and experiences rests on research approaches which differ from, but potentially complement, those that are otherwise 
dominant in SfD. In this final section we therefore reflect critically on the extent to which this more deliberately localized research approach can contribute to further development of knowledge and understanding of SfD.

The discussion that follows addresses two methodological dimensions that we believe can contribute to enhanced knowledge and understanding of SfD conducting what we have termed 'wide-lens' research to ensure specific SfD practices are contextualized, and engaging in locally informed collaborative knowledge production ('close-up' research). The chapter concludes with a final reflection on how these approaches may combine with established forms of SfD research to inform the development of SfD policy and practice.

\section{Wide-lens research: developing contextualized understandings}

One of our key aims in our research has been to understand SfD in context. We have therefore found it helpful to bring together findings from research that spans SfD policies, organizations, delivery practices and participant experiences. We are, however, conscious that this body of work is made up of separate studies; while they have multiple interconnections, they do not constitute a single cohesive research programme. The resulting 'jigsaw' of knowledge is wide-ranging but far from complete, and exhibits both strengths and weaknesses.

Undertaking these interlocking studies has allowed us to draw on contextual knowledge from one research project to inform another - for example, in our examination of the interaction of $\mathrm{SfD}$ with 'education', which has been addressed from policy level to the individual. We have conducted studies with school pupils and teachers on the use of sport to deliver HIV/AIDS education; with head teachers and pupils' parents on the role of sport in young people's lives; with government officials and international stakeholders on the position of sport in education policy and school curricula; with NGO staff, peer leaders and participants on the value of community-based provision; and with community leaders on the delivery and response of sport-based gender empowerment programmes. Together this data has offered greater insights than any single study into how the trajectories of education provision in Zambia interact with the localized experiences of individuals, and how this may impact the relationship between $\mathrm{SfD}$ and education.

Our research has also been characterized by fluidity; our knowledge and research approaches have evolved organically across several years. The development of the work conducted on HIV/AIDS illustrates this. The centrality of HIV/AIDS to the work of Zambian SfD organizations was emphasized to us 
from our very first visit, in 2006. It was Davies' knowledge of the scale and significance of other organizations' provision of HIV/AIDS education in Zambia that led to our investigation the following year of the extent of partnerships and integration between SfD and Zambia's broader HIV/AIDS sector. While undertaking this study, our need to better understand broader HIV/AIDS policy and the 'NGO world' led to greater engagement with the development studies literature. In turn, this influenced the design of Iain's 2009 study of community collaboration and Davies' PhD study of the mainstreaming of HIV/AIDS by NSAs. In some respects our knowledge has, therefore, grown substantially since the start of our studies, but still has clear limits.

Our efforts to contextualize SfD are also limited by the scale and selective coverage of our studies. Most of our research has been conducted in urban settings and our detailed work has largely been conducted with just two of the many SfD NGOs working in Zambia. Our studies have also involved a limited range of young people (again, a realization that has grown over time); work with programme participants and with school pupils has not included more severely excluded Zambian young people, such as those in rural areas. Some individual studies have also been constrained to the parameters prescribed by funders, but have benefitted from an extended three-to-five-year timescale; conversely, studies that have allowed us greater autonomy (for example, our investigation of integration and partnership with HIV/AIDS agencies in 2007) have been of short duration that has limited detailed tracking of trends over time. In valuing the way in which multiple studies can be combined to contextualize Zambian SfD, these limits must also be acknowledged.

We have found wide-ranging consideration of context to be fundamental to understanding Zambian SfD. This contrasts with the emphasis given within the evidence discourse to tightly specified units of knowledge that are framed by the positivist paradigm. Like Stanhope and Dunn (2011), we are unconvinced that social problems are amenable to this type of 'scientific process' or that its application to complex social problems can provide viable solutions (ibid.: 275276). The positivist methods used in such work produce data in which social problems are decontextualized and simplified (ibid.: 277); this clearly does not capture complex, diverse and individually and/or locally specific impacts that we have identified with Zambian SfD. In our view, methods that 'corral human behaviour into manageable measurable constructs' ( $i b i d$.) need to be challenged with vigour in SfD. Instead, SfD research needs to turn away from efforts to 'quantify the unquantifiable' (Pratt, 2015) and embrace much greater use of methods that capture complexity, difference and local specificity. 
How realistic is it for those conducting SfD research within the 'evidence' paradigm to incorporate this more sociologically driven approach? Funders of SfD typically require evaluators to work to narrow terms of reference, meet prescriptive data requirements, and report in management-focused and largely formulaic formats. This restricts methodological choice and evaluators rarely have the freedom to break free from such constraints. There may, nonetheless, be scope for some proportionate reorienting of methods used. In particular, we suggest:

- Scholarly enquiry does not need to be solely focused on programme specifics to be useful to programme deliverers; knowledge and understanding are also required. A shift away from the overemphasis on 'what works' as the goal of research in SfD would be beneficial.

- $\mathrm{SfD}$ is practised in a range of contexts and orientated towards a hugely diverse range of outcomes. There should be less emphasis on developing 'models' of SfD, which imply it will work in the same way in all contexts and for all outcomes. It is not sufficient to state that models will 'take account of context': it must be clear how this will be operationalized.

- Quantitative and qualitative methods are not mutually exclusive. A rebalancing of the quantitative-qualitative dichotomy is needed to allow these categories of data to be recognized as complementary rather than oppositional.

- In the hands of skilled practitioners, qualitative methods deliver in-depth, rigorous data; in contrast to quantitative tools, they allow researchers to directly identify and probe issues of accuracy, cultural specificity and truthfulness. Despite this, qualitative data has been erroneously dismissed by some SfD scholars - for example, as 'heartfelt narratives' (Hartmann and Kwauk, 2011: 285) or 'idealized testimonials' (Coakley, 2011:307) - in criticisms that do not appear to distinguish between 'journalistic' descriptive narratives developed for promotional purposes and qualitative data obtained within rigorous research processes. To ensure the value and credibility of such data, rigour and quality must be demonstrable and reported.

- Diverse qualitative methods should be employed to suit different research participants, including participatory and child-friendly methods and co-production of knowledge.

A 'wide-lens' approach does not, therefore, aim to reduce social phenomena to 'manageable units', but to reveal their breadth and complexity, recognize 
contextual factors, and illuminate the processes of SfD delivery and engagement. By accommodating use of both qualitative and quantitative methods, it also aligns SfD with other areas of development where both are employed to understand the cultural, social, political and institutional environments within which programmes operate (see the World Bank's 1998 expert workshop on integrating quantitative and qualitative research in development programmes, reported on in Bamberger, 2000). These approaches have led to innovation in design, data collection, and reporting methods and formats, such as the use of 'storybook' approaches to structure and report on the outcomes of participatory evaluation activities (e.g. Roughly and Dart, 2009). Moving closer to the approaches of the wider development sector may thus enhance both the quality and credibility of the SfD evidence-base.

A 'wide-lens' approach may also have some implications for critical-theoretical approaches to SfD. While often making use of qualitative methods, as yet much critical-theoretical SfD research tends not to underpin all levels of analysis empirically. There has been a tendency to foreground the perspectives of international stakeholders, with less coverage of those best positioned to give in-depth accounts of local contexts. Primary data, irrespective of being locally generated or otherwise, is viewed through a rather distant theoretical lens. Theoretical links between structural influences and local experiences are essential, but even the most insightful analyses may fall short without empirical investigation into how contextual factors play out locally. Following Long (2001), we have come to believe that knowledge about SfD can only exist within understandings of its particular contexts and be grounded in empirical, locally specific study. We therefore advocate alignment with empirically orientated development studies literature, and see empiricism as the bedrock of informed contextualized research.

\section{Close-up research: collaborative knowledge production}

A key aspect of our research has been our relationships with local actors, which have developed over time and across different studies. During our initial research we were interested in seeing how realistic undertaking research in this setting was, as predominantly white global-North researchers dropping into local communities. We became very aware of the limitations; on the one hand, Zambians involved in SfD were welcoming and forthcoming in discussing their communities, organizations and activities, and indeed their own lives; on the other hand, we were continually aware of the difficulties of establishing ourselves as independent from UK funding agencies. Even on visits when we were not 
undertaking research directly for UK agencies, it was necessary to continually emphasize our separation to avoid being viewed as a source of potential future income to be loaded only with 'happy stories'. Despite our efforts, undoubtedly we were presented with particular views and values that were considered appropriate for white global-North visitors. It would be naïve to suggest that this situation has been eliminated.

We have, however, now become something of a regular fixture within Lusaka; indeed, as we were working on this book in 2015, researchers on one of our projects returned from a visit to EduSport, due to continue to 2018. The long time over which contact has been maintained has contributed to the quality of our relationships with Zambian counterparts and, to some extent, counterbalanced the limited time periods we have usually spent in Zambia. We have known several staff of EduSport and Sport in Action, and some participants, since we first visited in 2006 and have spent time with them on every visit since. Boundaries between research work and more informal discussions and social activities have often blurred.

In comparison, formats and contexts for some particular data collection activities have inevitably been more structured and formal to varying degrees. However, as qualitative researchers asking young people and adults to talk openly about their home lives, families and communities, we have always expected to reciprocate. At the end of our interviews and focus groups about empowerment, poverty and experiences of HIV, we have habitually made the offer: 'Is there anything you would like to ask us about now, about the research or about our lives back in England?' In response we have been questioned about anything from whether we are Manchester United supporters to how we balance work and family; Tess and Ruth especially have been asked about their home lives and children. In many cases the knowledge exchanged has been quite personalized.

These experiences raise some questions about prevailing discourses that emphasize the need for distance in relationships between researchers and 'the researched' in SfD. Rationalistic, positivist approaches are predicated upon the notion of the detached researcher. UK Sport's 2006 'Sport-in-Development: A Monitoring and Evaluation Manual, for example, stipulates that quantitative data collection should be undertaken by people who are not related to the programme:

The interviewing should not be undertaken by personnel directly involved in delivering the programme. Where possible, a neutral person(s) not known to 
the participants should be used to reduce the potential for biased responses. (Coalter, 2006: 51, emphasis as in original)

It is standard for quantitative studies to avoid closeness between researchers and respondents, which is considered to compromise the supposed 'objectivity' of the method. It is less certain that detachment automatically produces superior knowledge and understanding compared to qualitative data collection characterized by qualities of empathy and trust. The use of qualitative approaches may require some SfD scholars to reconsider their epistemological positioning.

Our work in Zambia has increasingly been based on familiarity and trusting relationships, and characterized not by detachment but by collaboration and reciprocal learning. Our experience has been that this building of relationships over time with SfD NGOs, their staff and young people has not led to collusion, but to greater openness. Those we have spoken to have been less concerned to mask negative issues; staff have talked more about the challenges and frustrations of their work, and peer leaders about the limits to their impact. Being told about these difficulties has allowed us to make suggestions for how they might be addressed, and our research has therefore been felt to be of practical value to their work. This has enabled all of us - researchers, staff from SfD NGOs and funders - to adopt a more critical stance than had previously been possible; to come closer to an understanding of how much or how little sport may offer development; and to learn more about the forms of support required to achieve this. Our being involved researchers has, therefore, enhanced the quality of the research, not detracted from it.

Underpinning this approach has been our commitment to localizing and decolonizing knowledge production. The decolonization standpoint advocates that knowledge production can significantly benefit from culturally appropriate ownership and leadership across all stages of the research process. This clearly contrasts with research 'into' the global South that has been designed, implemented and reported by global North researchers. Instead, it favours local participation in knowledge production, in which research agendas and focus are derived from local interests; research design reflects local forms of communication; data collection methods use appropriate tools, language and concepts; and reporting identifies findings and guidance that address local priorities, presented in formats that are usable by local actors. These points therefore address important details of method and methodology, and have practical implications; for example, they direct researchers to use qualitative in-depth interviews among communities with strong oral traditions, and to 
avoid written tools among groups with low literacy. One of our own lessons in this respect has been that local data collection can allow participants whose language has no written form to participate in our research; for example, in the most recent phases of our work with the Go Sisters programme, we have supported peer leaders to conduct interviews with Nyanja-speaking parents in communities.

The decolonization discourse has considerable acceptance within development studies and has been advocated by those in the critical-theoretical strand of SfD literature. However, the relative lack of empirical application of such approaches has limited the extent to which decolonized research has been accepted in SfD. Its wider adoption may be hindered by its emphasis on localized, culturally specific and reflexive practices which do not sit well with those who align with the evidence discourse and advocate rigid application of standardized research designs and tools. We would suggest that $\mathrm{SfD}$ research has now reached a stage of maturity where it can - and needs to - move beyond defensiveness to engage in more constructive critical and realistic self-evaluation of its processes of knowledge production.

\section{Localizing $\mathrm{SfD}$ in research and in practice}

Knowledge production, by researchers and evaluators, is vital to development policy and practice. Across the different instrumental and critical-theoretical strands of SfD research, alternative claims are made for their (potential) contributions to improving SfD practices and policies. Critical theorists have called for significant realignments towards more radical SfD approaches. Hartmann and Kwauk (2011: 293), for example, advocate such an approach, in which:

Actors would be empowered to participate critically in the transformation of not only their own experiences in society but also of the world itself through a collective resistance against the hegemonic structures and relations of inequality that get reproduced through sport.

Conversely, those who alternatively subscribe to rationalistic evaluation claim that their approach offers a route, and perhaps the only route, to the improvement of specific programmes. Richards et al's opening editorial for the Journal of Sport for Development is a case in point:

SFD organizations must 'evaluate or perish'. Only by applying rigorous research methods will the SFD sector establish adequate evidence to streamline its 
approach and survive broad contractions in foreign aid budgets. (Richards et al., 2013: 2)

We avoid these overarching suggestions for alternative approaches to SfD as well as any attempt, within a book of this nature, to identify specific modifications that may be made by Zambian SfD organizations. While elements of the radical approaches suggested by Hartmann and Kwauk (2011) and others are identifiable within Zambian SfD, as we have also shown, there remain considerable barriers to their widespread or wholesale adoption. Nevertheless, to advocate and utilize decolonized qualitative research is not to 'abjure programme improvement', as Coalter (2013: 58) appears to suggest. Rather, we would argue that in-depth, contextually grounded and localized understandings, such as that developed throughout this book, are essential to any efforts to improve SfD practices or policies. The contributions of the types of understandings that we have developed may not be as direct, specific, overarching or radical as others would suggest. However, without such understandings, attempts towards improvement may well founder upon misdiagnosis and misapplication.

Our experiences of working in Zambia over a long period of time have led us to believe that understanding in SfD requires more detailed, wide-ranging knowledge of context than is habitually collected, and needs knowledge about local context especially. Perhaps counter-intuitively, the need to open up to the wider sociological perspective is especially relevant to evaluation studies. Data that measures 'performance' is not sufficient to inform efforts to deliver social change through sport. In this vein, Pratt (2015) has recently dismissed as 'an intellectual farce' the production of evidence that is driven by bureaucratic and political requirements for clarity and simplicity rather than a desire to reflect the complex reality of development. He especially criticized the persistence of 'formulaic evaluations' that attempt to '[force] data into a pre-set framework rather than allowing it to speak for itself', resulting in 'evaluating people against objectives imposed by a funder or negotiated by a fundraiser, but never agreed by those actually running the programme.

We remain aware and concerned about the limitations of the research approaches and data we have presented in this book. We are especially conscious of our own dominance in this account; as much as we have attempted to diffuse the inherent power relations between global-North researchers working with global-South participants, these have inevitably shaped our discussions and data. We also recognize that our attempts to draw together our 
many projects may well produce a more piecemeal picture than we would hope. We nonetheless hope that this attempt to connect these different snapshots may signal the potential for a more holistic understanding of different dimensions of SfD. Localizing global SfD is not only about knowledge production; whether they use sport or not, efforts to improve the lives of young people will necessarily be limited without deep understanding of their local realities. 